\title{
Sonazoid-Enhanced Ultrasonography and Ga-EOB-DTPA-Enhanced MRI of Hepatic Inflammatory Pseudotumor: A Case Report
}

\author{
Masaya Saito ${ }^{1}$, Yasushi Seo ${ }^{1}$, Yoshihiko Yano ${ }^{1,2}$, Akira Miki ${ }^{1}$, Yukiko Morinaga ${ }^{3}$, \\ Tomoo Itoh ${ }^{3}$, Masaru Yoshida ${ }^{1,4}$ and Takeshi Azuma ${ }^{1}$
}

\begin{abstract}
A liver neoplasm was found in a 63-year-old man with alcoholic liver disease. Sonazoid-enhanced ultrasonography (US) showed that the neoplasm was isoechoic at the early vascular phase and hypoechoic at the post-vascular phase. Gadolinium ethoxybenzyl-diethylenetriamine-enhanced magnetic resonance imaging (MRI) showed that the neoplasm was hypointense at the hepatobiliary phase. We suspected that it was a malignant tumor. By needle biopsy, however, the neoplasm was diagnosed as an inflammatory pseudotumor (IPT). We encountered a rare case of hepatic IPT, the differential diagnosis of which was difficult to distinguish from malignant tumor. Here, we report new US and MRI findings of hepatic IPT.
\end{abstract}

Key words: inflammatory pseudotumor, sonazoid-enhanced ultrasonography, gadolinium-ethoxybenzyldiethylenetriamine, magnetic resonance imaging, computed tomography

(Intern Med 51: 723-726, 2012)

(DOI: 10.2169/internalmedicine.51.6811)

\section{Introduction}

Inflammatory pseudotumor (IPT) is a rare benign neoplasm found in almost every organ of the human body $(1,2)$. Involvement of the liver is rare (3). Recently, new imaging modalities for the diagnosis of liver neoplasm have been improved and hepatic IPT is becoming detectable (4). Hepatic IPT can radiologically resemble various malignant tumors. On dynamic computed tomography (CT) scans, the hepatic IPT was reported to show a pattern of enhancement of metastatic tumor or hepatocellular carcinoma (HCC) (4). However, the enhancement patterns of gadolinium-ethoxybenzyl-diethylenetriamine ( Ga-EOBDTPA)-enhanced magnetic resonance imaging (MRI) on hepatic IPT have not been reported. This paper is the first to report the enhancement pattern of Ga-EOB-DTPA-enhanced MRI on hepatic IPT.
Most of the previously reported cases were preoperatively misdiagnosed as malignant tumors and were correctly diagnosed after surgical resection (5-7). Very few reported cases have been diagnosed by needle biopsy (5). It is important to diagnose IPT on liver biopsy because it can be cured with medical treatment including nonsteroidal anti-inflammatory drugs, antibiotics or steroids (3). In addition, spontaneous regression or regression after conservative treatment has been reported $(1,2,5,7,8)$. In the present case, we performed fine needle aspiration biopsy of the neoplasm and were able to diagnose it as an IPT.

\section{Case Report}

The patient was a 63-year-old man with no chief complaint. He was a heavy drinker, consuming over $30 \mathrm{~g}$ of alcohol per day from 20 years of age. He had hyper-urinary acidemia, alcoholic liver disease and renal stone from 60

\footnotetext{
${ }^{1}$ Division of Gastroenterology, Department of Internal Medicine, Kobe University Graduate School of Medicine, Japan, ${ }^{2}$ Center for Infectious Diseases, Kobe University Graduate School of Medicine, Japan, ${ }^{3}$ Division of Diagnostic Pathology, Department of Pathology, Kobe University Graduate School of Medicine, Japan and ${ }^{4}$ Division of Metabolomics Research, Department of Internal Medicine, Kobe University Graduate School of Medicine, Japan

Received for publication October 26, 2011; Accepted for publication December 12, 2011

Correspondence to Dr. Takeshi Azuma, azumat@med.kobe-u.ac.jp
} 

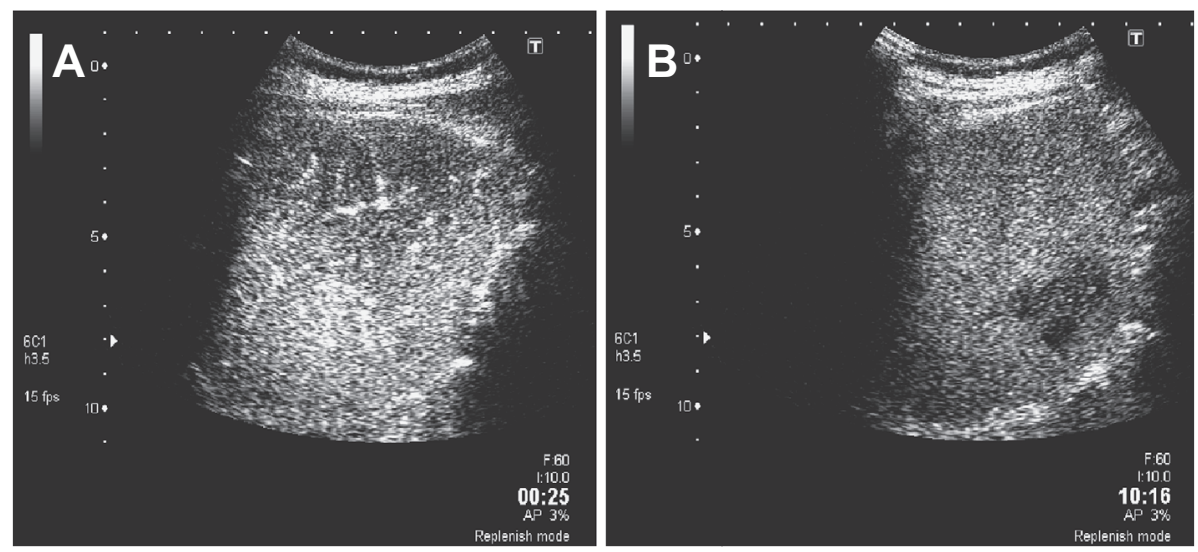

Figure 1. Sonazoid-enhanced US of hepatic inflammatory pseudotumor (IPT). (A) The IPT was isoechoic at the early vascular phase (within 1 minute after intravenous injection of sonazoid). (B) It was hypoechoic at the post-vascular phase (more than 10 minutes after the injection). The enhancement patterns resembled the pattern of a hypovascular tumor.

years of age. He did not have hypertension, diabetes mellitus or hypercholesterolemia, and had no family history including liver diseases. He was followed-up by biannual blood tests and abdominal US or CT. In 2010, a single lowdensity region $30 \mathrm{~mm}$ in diameter was detected in liver segment 8 by abdominal plain CT scans. Therefore, he was admitted to our hospital for further evaluation. On admission, his body temperature was $36.3^{\circ} \mathrm{C}$ and body weight was 67.5 $\mathrm{kg}$, without weight loss during the previous 6 months. The abdomen was soft and flat. Bowel sounds were normoactive. The liver was not palpable, without tenderness. The blood test results on admission were as follows: white blood cells, $4,300 / \mu \mathrm{L}$; hemoglobin, $14.0 \mathrm{~g} / \mathrm{dL}$; platelet count, $17.1 \times 10^{4} /$ $\mu \mathrm{L}$; prothrombin time, $>100 \%$; C-reactive protein, $0.3 \mathrm{mg} /$ $\mathrm{dL}$; albumin, $3.8 \mathrm{~g} / \mathrm{dL}$; total bilirubin, $0.7 \mathrm{mg} / \mathrm{dL}$; aspartate aminotransferase, $36 \mathrm{IU} / \mathrm{L}$; alanine aminotransferase, $38 \mathrm{IU} /$ L; lactate dehydrogenase, 206 IU/L; alkaline phosphatase, 205 IU/L; $\gamma$-glutamyltranspeptidase, 79 IU/L; choline esterase, $278 \mathrm{IU} / \mathrm{L}$; HBs Ag, (-); HBs Ab, $145 \mathrm{mIU} / \mathrm{mL}(+)$; $\mathrm{HBc} \mathrm{Ab}, 1.79$ (+); HCV Ab, (-); alpha-fetoprotein, $5 \mathrm{ng} / \mathrm{mL}$; des-gamma-carboxy prothrombin, $19 \mathrm{mAU} / \mathrm{mL}$; carcinoembryonic antigen, $3.6 \mathrm{ng} / \mathrm{mL}$; carcinogenic antigen 19-9, 27 $\mathrm{U} / \mathrm{mL}$; and interleukin-2 receptor, $391 \mathrm{U} / \mathrm{mL}$. Upper and lower endoscopy showed no significant findings. Abdominal dynamic CT scans showed that the periphery of the neoplasm was slightly enhanced at the delayed phase (data not shown). Sonazoid-enhanced US showed that the neoplasm was isoechoic at the early vascular phase (within 1 minute after intravenous injection of sonazoid) and hypoechoic at post-vascular phase (more than 10 minutes after injection) (Fig. 1A, B). MRI displayed that the neoplasm was entirely hypointense on the unenhanced T1-weighted image, partially hyperintense on the unenhanced T2-weighted image and entirely hypointense at the hepatobiliary phase of Ga-EOBDTPA-enhanced image (Fig. 2A-C). Therefore, we suspected that the neoplasm was a malignant tumor. We recommended surgical resection for our patient, but he refused it. We performed fine needle aspiration biopsy of the neoplasm as a diagnostic tool in place of surgical resection. The tissue specimen showed marked infiltration of plasma cells, which were surrounded with dense fibrous connective tissue (Fig. 3A, B). However, lymph follicle formation was not observed (Fig. 3A). From these pathological findings, we diagnosed the tumor as an IPT. Two months after the diagnosis, the diameter of the IPT spontaneously shrank from $30 \mathrm{~mm}$ to $25 \mathrm{~mm}$ (data not shown).

\section{Discussion}

Hepatic IPT, first described by Pack and Baker in 1953, is a rare benign liver disease characterized by a wellcircumscribed mass of chronic inflammatory cell infiltration and fibrosis (9). Hepatic IPT is predominantly seen in men and the reported mean age of the patients is 37 years old (10). Most of the reported cases involve solitary masses, and they measure up to $2.5 \mathrm{~cm}$ in diameter. The present patient was a 63-year-old man, and the IPT was solitary and $3.0 \mathrm{~cm}$ in diameter. Some reports have shown that the IPT was not a disease entity, but rather a group of solidappearing lesions that grossly mimic tumors, as in the present case (3).

The etiology of hepatic IPT remains unclear, although infection, biliary obstruction, chronic cholangitis (11), and primary sclerosing cholangitis (12) have all been suggested to be possible causes. All previously reported cases had one or more symptoms, such as fever, epigastric pain, vomiting, general malaise, or weight loss. Among these symptoms, a high fever was the most frequent symptom. In the present case, however, the physical examinations, laboratory data, and imaging suggested none of the possible causes such as biliary obstruction, cholangitis, and chronic inflammation, and our case did not present any symptoms. Therefore, it was difficult to speculate the etiology of IPT in this case.

As for the radiological findings of hepatic IPT, the present case showed delayed enhancement of the periphery was observed on CT scans. This finding can be explained as an 

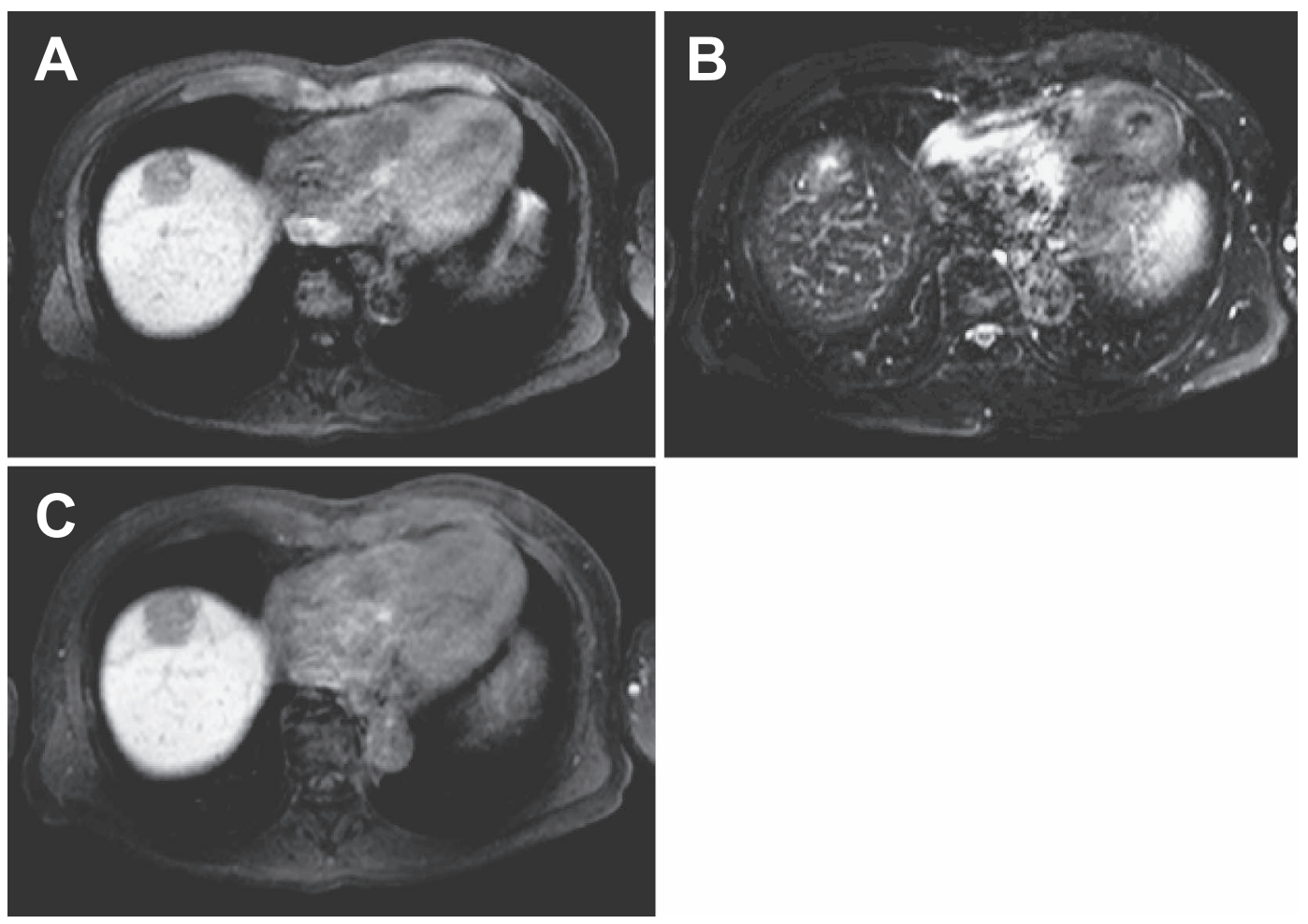

Figure 2. Ga-EOB-DTPA-enhanced MRI of hepatic inflammatory pseudotumor (IPT). (A) The IPT was entirely hypointense on the unenhanced T1-weighted image, (B) partially hyperintense on the unenhanced T2-weighted image, and (C) entirely hypointense on the hepatobiliary phase of GaEOB-DTPA-enhanced image. The enhancement patterns also resembled the pattern of a hypovascular tumor.
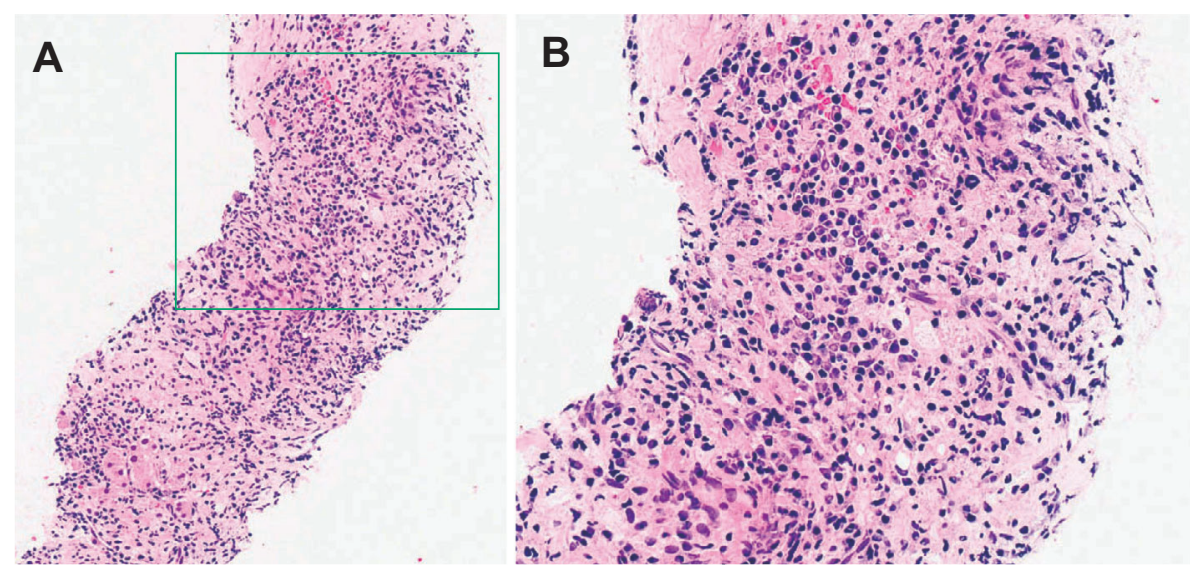

Figure 3. Histopathological findings of the needle biopsy. The tissue specimen showed fibrosis (A: Hematoxylin and Eosin staining, $\times 100)$ and marked infiltration of plasma cells $(\mathrm{B}$ : Hematoxylin and Eosin staining, $\times 200$ ). Lymph follicle formation was not observed (A). From these pathological findings, we diagnosed IPT. B is a magnification of the square area designated in A.

accumulation of extravascular contrast media in fibrotic components within the mass $(13,14)$. Variable degrees of fibrosis and cellular infiltration can cause diverse manifestations on images of IPT. In addition, our case did not show liquefactive necrosis of the liver parenchyma, appearing as an area with the density of water on CT scans, so we can differentiate the IPT from a liver abscess, the other representative disorder of inflammatory liver diseases (15).
Hepatic IPT sometimes mimics malignant tumors, such as well-differentiated hepatocellular carcinoma, metastatic liver tumors or cholangiocarcinoma. Previous studies have noted that delayed-phase CT and fluorodeoxyglucose positron emission tomography do not distinguish hepatic IPT from malignant tumors $(16,17)$. The present case is the first case reported in which the IPT was hypodense at the hepatobiliary phase of Ga-EOB-DTPA-enhanced MRI. This finding is 
also similar to those of malignant tumors, so it may be difficult or impossible to differentiate IPT from these tumors.

In most of the prevsiously reported cases, the patient underwent surgical resection due to difficulties in making a preoperative diagnosis. Only a few cases have actually been treated conservatively after a diagnosis of IPT confirmed by percutaneous biopsy (12). The present patient was a rare case that was diagnosed by needle biopsy. Percutaneous biopsies are helpful for making a correct diagnosis.

Histologically, hepatic IPT is categorized into three types: xanthogranuloma type, plasma cell granuloma type, and hyalinized sclerosing type (18). In the present case, striking infiltration of innumerable numbers of plasma cells was seen, so it was categorized as plasma cell granuloma type. However, a previous study showed that histological appearance was not correlated with the disease stage (3). Moreover, it was shown that these various morphological features were observed to some extent simultaneously even in the same case (3). Therefore, morphological classification of IPT did not appear to be practically valuable.

The prognosis of patients with hepatic IPT is generally excellent. Adequate therapy for IPT is considered to be conservative treatment with an antibiotic agent, not surgical resection. In the present case, only observation was conducted, without any medication. Two months after the establishment of the diagnosis, the IPT shrank spontaneously from $30 \mathrm{~mm}$ to $25 \mathrm{~mm}$ in diameter on the repeated CT scans. The repeated imaging studies of the IPT indicated its spontaneous regression. Such studies can also play a key role in making a correct diagnosis of IPT.

In conclusion, the present case is the first for which the enhancement patterns of Ga-EOB-DTPA-enhanced MRI in hepatic IPT have been reported. In addition, our case was a rare example of hepatic IPT with a difficult differential diagnosis to distinguish it from a malignant tumor.

\section{The authors state that they have no Conflict of Interest (COI).}

\section{Acknowledgement}

We thank the nurses and our colleagues in the Department of Gastroenterology and the Gastroenterology Ward at Kobe University Hospital for their cooperation. This work was supported by grants for the Global COE Program, Global Center of Excellence for Education and Research on Signal Transduction Medicine in the Coming Generation from the Ministry of Education, Culture, Sports, Science, and Technology of Japan [T.A and M. Y].

\section{References}

1. Kim YW, Lee JG, Kim KS, et al. Inflammatory pseudotumor of the liver treated by hepatic resection: a case report. Yonsei Med J 47: 140-143, 2006.

2. Andrade DM, Martins SJ, Paz O, Cardozo JB, Novses AE, Santiago MB. Inflammatory pseudotumor: a diagnostic dilemma. Eur $\mathbf{J}$ Intern Med 17: 514-516, 2006.

3. Horiuchi R, Uchida T, Kojima T, et al. Inflammatory pseudotumor of the liver: clinicopathological study and review of the literature. Cancer 65: 1583-1590, 1990.

4. Tsou YK, Lin CJ, Liu NJ, et al. Inflammatory pseudotumor of the liver: Report of eight cases, including three unusual cases, and a literature review. J Gastroenterol Hepatol 22: 2143-2147, 2007.

5. Schnelldorfer T, Chavin KD, Lin A, et al. Inflammatory myofibroblastic tumor of the liver. J Hepatobiliary Pancreat Surg 14: 421423, 2007.

6. Locke JE, Choti MA, Torbenson MS, et al. Inflammatory pseudotumor of the liver. J Hepatobiliary Pancreat Surg 12: 314-316, 2005.

7. Yamaguchi J, Sakamoto Y, Sano T, et al. Spontaneous regression of inflammatory pseudotumor of the liver: report of three cases. Surg Today 37: 525-529, 2007.

8. Lo OS, Poon RT, Lam CM, et al. Inflammatory pseudotumor of the liver in association with a gastrointestinal stromal tumor: a case report. World J Gastroenterol 10: 1841-1843, 2004.

9. Pack GT, Baker HW. Total right hepatic lobectomy: Report of a case. Ann Surg 138: 253-258, 1953.

10. Shek TW, Ng IO, Chan KW. Inflammatory pseudotumor of the liver. Report of four cases and review of the literature. Am J Surg Pathol 17: 231-238, 1993.

11. Nakanuma Y, Tsuneyama $K$, Masuda $S$, et al. Hepatic inflammatory pseudotumor associated with chronic cholangitis. Hum Pathol 25: 86-91, 1994.

12. Toda K, Yasuda I, Nishigaki Y, et al. Inflammatory peudotumor of the liver with primary sclerosing cholangitis. J Gastroenterol 35: 304-309, 2000.

13. Yoshikawa J, Matsui $\mathrm{O}$, Kadoya $\mathrm{M}$, et al. Delayed enhancement of fibrotic areas in hepatic masses: CT-pathologic correlation. J Comput Assist Tomogr 16: 206-211, 1992.

14. Itai Y, Ohtomo K, Kokubo T, et al. CT of hepatic masses: significance of prolonged and delayed enhancement. AJR 146: 397-402, 1986.

15. Halvorsen RA, Korobkin M, Foster WL, et al. The variable CT appearance of hepatic abscesses. Am J Roentgenol 141: 941-946, 1984.

16. Fukuya $T$, Honda $H$, Matsumata $T$, et al. Diagnosis of inflammatory pseudotumor of the liver: value of CT. Am J Roentgenol 163: 1087-1091, 1994.

17. Kawamura E, Habu D, Tsushima $\mathrm{H}$, et al. A case of hepatic inflammatory pseudotumor identified by FDG-PET. Ann Nucl Med 20: 321-323, 2006.

18. Someren A. "Inflammatory pseudotumor" of liver with occlusive phlebitis: report of a case in a child and review of the literature. Am J Clin Pathol 69: 176-181, 1978.

(C) 2012 The Japanese Society of Internal Medicine

http://www.naika.or.jp/imindex.html 